\section{Cahiers de Narratologie}

Analyse et théorie narratives

8 | 1997

Création de l'espace et narration littéraire

\title{
Poésie et espace chez Guillevic
}

\section{Colette Guedj}

\section{OpenEdition}

Journals

Édition électronique

URL : http://journals.openedition.org/narratologie/10746

DOI : 10.4000/narratologie.10746

ISSN : 1765-307X

Éditeur

LIRCES

Édition imprimée

Date de publication : 1 décembre 1997

Pagination : 141-156

ISBN : $291089746 X$

ISSN : 0993-8516

Référence électronique

Colette Guedj, «Poésie et espace chez Guillevic », Cahiers de Narratologie [En ligne], 8| 1997, mis en ligne le 01 décembre 2020, consulté le 25 février 2021. URL : http://journals.openedition.org/ narratologie/10746 ; DOI : https://doi.org/10.4000/narratologie.10746

Ce document a été généré automatiquement le 25 février 2021.

Article L.111-1 du Code de la propriété intellectuelle. 


\section{Poésie et espace chez Guillevic}

\section{Colette Guedj}

1 Guillevic est par excellence le poète de l'espace, un espace qui loin d'être évanescent ou éthéré est au contraire celui d'un homme solidement arrimé au réel. Les titres des recueils, - et des poèmes - sont édifiants : des mots qui désignent la matière concrète et dure: Murs, Pierres, Pétrée mais qui ressortissent aussi au geste (du sculpteur) d'en briser l'opacité abrupte, de la creuser, de l'entamer, de pénétrer à l'intérieur du monde (Inclus, Avec).

2 Ce que je me propose de montrer ici, c'est la récurrence thématique de la minéralité qui structure le rapport de Guillevic à l'espace, un rapport qui relève moins de la géographie (cependant bien présente avec Carnac) que d'une attitude mentale propre à l'homme et au poète. En second lieu j'évoquerai le rôle de l'espace (autrement dit du blanc) en tant que facteur structurel et rythmique de cette écriture. J'illustrerai mon propos de quelques figures qui fourniront ses repères visuels à ce parcours de l'espace guillevicien, que j'emprunterai au recueil Euclidiennes, texte qui consacre, non sans humour, l'alliance entre la poésie et l'abstraction: Guillevic y poursuit, en effet, un dialogue avec (pas moins de) cinquante deux figures géométriques, leur donnant tantôt la parole, tantôt les posant dans une neutralité distante, tantôt enfin se décrivant luimême, dans l'acte de création poétique, à travers elles. Ce livre d'images, à mi-chemin entre le manuel de géométrie et le livre d'enfants orné de figures me paraît, en tout état de cause, pouvoir symboliser de façon emblématique la poésie de Guillevic, et je 
l'envisagerai donc ici moins pour ce qu'il dit que pour ce qu'il nous donne à voir de cette poétique, - en exaltant sa valeur testimoniale en quelque sorte.

\section{perpendiculaire}

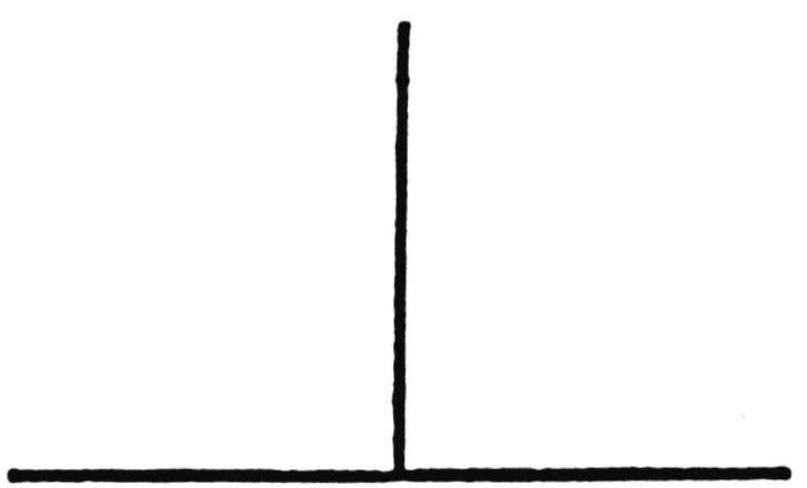

Facile est de dire

Que je tombe à pic.

Mais c'est aussi sur moi

Que l'autre tombe à pic.

Figure 1

trapèze

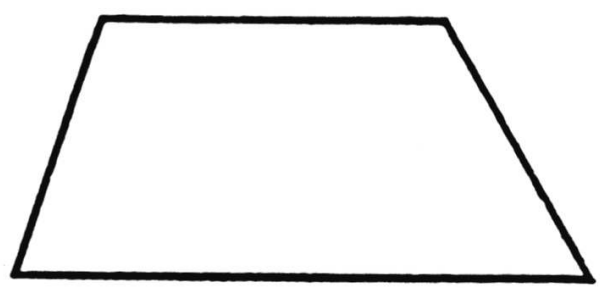

Qui mieux que moi

Parle surface pure et simple?

Qui mieux que moi

Ne parle de rien d'autre?

Ainsi j'invite

A parcourir,

A demeurer,

A longer, si l'on veut, mes bords,

A voir plus loin

S'il y a quelque chose à voir.

Figure 2 


\section{L'écriture de l'espace}

3 L'espace minéral et vertical, tel est l'un des thèmes fondateurs de la poésie guillevicienne, que je qualifierais d'existentiel et d'ontologique, car s'y s'inscrit une expérience du monde et de l'être (ou plutôt de l'être au monde). Espace dur de la pierre, du granit, de la matière solide, qui s'accorde avec le matérialisme du poète. Espace compact et dense que symbolise l'un des mots-clefs de sa poésie, celui de la paroi. (Figure 1 : " perpendiculaire ») qui, par la résistance qu'elle oppose à l'homme, le renvoie à l'existence de son propre corps, l'empêchant de se déliter (une hantise chez Guillevic, sur laquelle je reviendrai). Car le poète existe, non seulement que parce qu'il est séparé des choses, parce qu'elles lui résistent:

« Transgresser, franchir

Aller plus loin,

Ailleurs, toujours,

Cogner, se cogner »

(Paroi)

mais surtout parce que ces choses lui offrent le contour fini de leur surface lisse (Figure $2:$ « trapèze »), opposée à la profondeur qu'il redoute et le terrifie :

« L'armoire était de chêne

Et n'était pas ouverte.

Peut-être il en serait tombé des morts,

Peut-être il en serait tombé du pain.

Beaucoup de morts

Beaucoup de pain $"$.

(Terraqué),

Ou encore :

«Voir le dedans des murs

Ne nous est pas donné

On a beau les casser

Leur façade est montrée "

(Exécutoire)

En revanche l'eau, celle de l'étang qui a une présence obsessionnelle dans l'œuvre, est inquiétante parce que sous l'apparence placide de sa surface, elle contient la profondeur glauque et visqueuse qui menace l'intégrité du moi. L'étang est ainsi toujours lié chez Guillevic au mauvais rêve, et au meurtre (symbolique)ํ․

7 Les exemples sont légion de ce rêve du poète de faire corps avec le roc $^{2}$ pour faire échec à la béance de l'univers :
« Être paroi
Se confondre
Avec la paroi
L'intégrer
S'intégrer
Rêver le temps
Devenu corps".

pour conjurer le temps, à l'instar du « Rocher » :

«J'ai besoin d'être dur

Et durable avec toi ;

Contre tout l'ennemi

Que ta surface arrête,

Besoin que nous soyons 
Complices dans la veille

Et la nuit passera

Sans pouvoir nous réduire ».

(Sphère)

Le rocher, le roc, le menhir (autant de termes récurrents dans sa poésie), le poète leur envie leur espace fermé, - et circonscrit :

«Mais c'est bon pour les rocs

D'être seuls et fermés

Sur leur travail de nuit

Et peut-être qu'ils savent

Vaincre tout seuls leur fièvre

Et résister tout seuls »

(Avec)

L'ambivalence est là cependant : car si l'espace dur de la minéralité confirme le poète dans l'existence de son corps, il le confirme aussi dans sa séparation d'avec le monde, autant dire dans sa solitude, une solitude - et c'est un élément capital - qu'il recherche et redoute tout à la fois. Il la recherche car elle est un élément de perpétuel ressourcement à soi-même, mais il la redoute car entre le fait d'être séparé des autres et celui d'être exclu il n'y a qu'un pas et c'est là l'une des hantises de Guillevic les plus taraudantes : celle très exactement de n'être pas inclus (cf. les recueils intitulés Avec, Inclus). Cette souffrance d'être rejeté au dehors, il la dit dans ce poème dont on aurait beau jeu de faire la psychanalyse (il confiera à maintes reprises, l'enfant mal aimé qu'il a été, laid, portant d'épaisses lunettes et délaissé au profit du frère mort que sa mère lui préféra toute sa vie) :

«Mère aux larmes brûlantes, l'homme fut chassé de vous

De vos tendres ténèbres,

(Terraqué) De votre chambre de muqueuses »

Figure 3 : «sphère 1 et 2 "

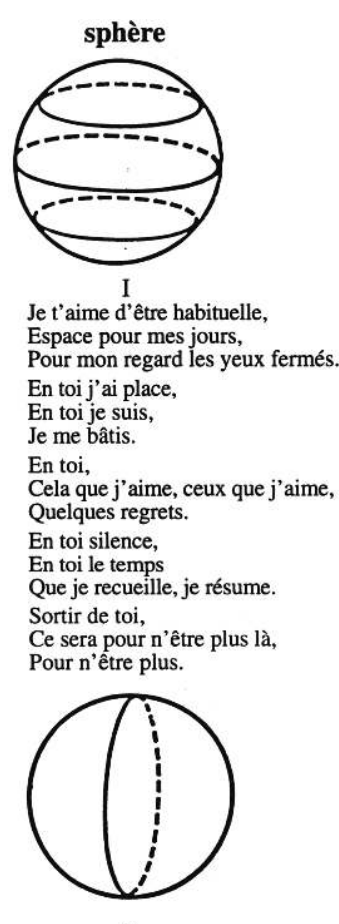

II

Et quand il n'y aurait

Que nous deux pour durer.
Quand il 'y aurait

Figure 3 
Cette souffrance ontologique de l'exclusion, et tragiquement anecdotique, se conjugue avec le sentiment de la solitude à laquelle il ne peut échapper, étant séparé du monde, mais qui le nourrit, et où il se complaît. D'où ce narcissisme de Guillevic, symbolisé par la figure du centre que le poète ne cessera de revendiquer : « Je suis au centre. Je ne suis pas un individu dans la société. Ce n'est pas du tout une question d'orgueil. J'ai besoin d'un centre. Si ce n'est pas moi, où est le centre? Le centre c'est moi. Tout part de moi $»^{3}$. Un narcissisme cependant bien singulier (j'y reviendrai) en ce sens qu'il englobe l'attitude lyrique tout en la rejetant; Guillevic, qui sait admirablement parler de sa poésie, résume cet aspect paradoxal du processus poétique en une formule saisissante : «En moi il n'y a pas de je » (Etier). Ou encore, de façon plus ludique, mais non moins sérieuse :

"Quand il écrit le pronom : Il

Au début d'une vers,

On dirait assez souvent

Qu'il a fait un Je,

Car le I devient grand comme un J

Et le i petit comme un e.

Donc Il

N'est pas forcément un autre.»

(Qui)

11 Je parlais d'ambivalence, mais mieux vaudrait dire contradiction, car ce désir fécond de se replier sur lui-même ne va pas sans celui de répondre, dans une ferveur inspirée, quasi mystique, à l'appel du monde. "Ma poésie est solidaire, elle est avec »" Étrange parenté paronymique entre "solitaire » et "solidaire ", déjà pointée par Camus dans un autre contexte, et qui en tout état de cause, relève certainement moins d'un télescopage - facile - entre les mots, que de l'attitude du poète qui dans le même temps qu'il s'ouvre au monde se ressource à son propre miroir. Il y a en effet chez Guillevic le désir d'être à l'unisson du monde dans une sorte de vision animiste et panthéiste, - dont il nous dit qu'elle lui vient davantage de son appartenance bretonne que chrétienne : «Être né au pays des menhirs - du monde mégalithique ces menhirs qui appartiennent à une civilisation dont on ignore tout et qui date de longtemps avant les Celtes. On est en plein inconnu, en plein mystère. On est dans le sacré. $»^{5}$.

Guillevic a un rapport charnel avec la terre, les genêts, l'eau, la lande, la rivière :

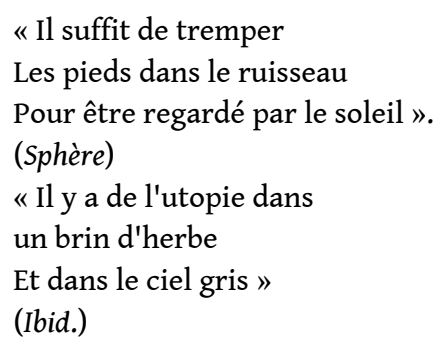

Le poète parle de ses rapports avec la terre (de son enfance) comme d'une célébration rituelle où la fête est capitale, et capitale surtout l'intrusion du sacré dans le quotidien : « Le rôle du poète, écrit-il, est de donner à vivre le sacré. A la limite poésie et sacré se confondent.[...] Le poète parce qu'il est l'homme du langage, a un rôle privilégié dans cette invention perpétuelle. [...] Dans les sociétés primitives il n'y avait pas le profane et le sacré, tout était sacré : manger, marcher, dormir [...]. Le poète doit aider les autres à vivre le sacré dans la vie quotidienne $»^{6}$. Célébrer les rites pour Guillevic revient très 
exactement à maintenir sa participation avec l'univers, et par voie de conséquence la nécessaire porosité entre le profane et le sacré :

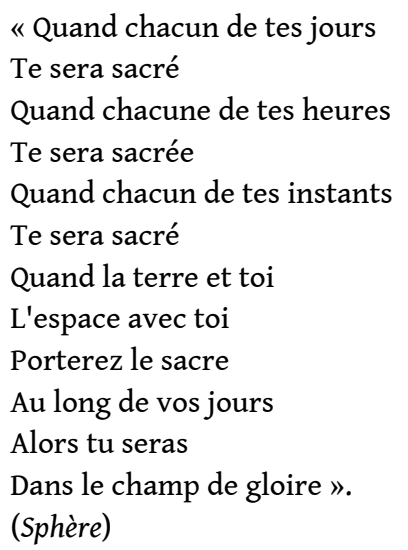

14 Le ton du poème est celui que l'on retrouvera dans de grands textes comme Requiem ou Magnificat ; de distique en distique, comme on dirait de degré en degré, on s'élève vers une sorte d'hymne à la nature, rituel et quasi hiératique. Le poème, en somme, comme cérémonial, comme rite d'initiation?

Cette tension permanente entre l'au-dehors et l'au-dedans chez Guillevic (et n'est pas aussi sans être source de joie pour le poète) explique très certainement la persistance des figures de l'entre-deux, celles du seuil, de la frontière, de la marge qui séparent et lient tout à la fois : on pense à certains titres de recueils, Avec, Déjà, Qui, Si, qui pour être de simples ligatures grammaticales n'en sont pas moins signifiantes, mais aussi à ces figures majeures de la poétique guillevicienne, telles que Terraqué (enserré de terre et d'eau) ou encore l'Étier (petit canal qui relie la mer aux marais salants) qui, par l'espace interstitiel qu'elles dessinent entre l'ouvert et le fermé (à l'instar du blanc dans le poème, sur lequel je reviendrai) garantissent l'homme du danger de délitement, autrement dit de celui de se dissoudre, de s'engluer, de perdre son identité. C'est la raison pour laquelle des mots comme "tourbillon » ou "nuage » ont toujours chez Guillevic des connotations défavorables: la marche des nuages, dit le poète, est la " marche des victimes ", de troupeaux qui n'ont pas

«Pour eux la chance

Qu'ont les murs de pierre

Qui peuvent peser

Ce qui leur advient » (Avec)

On retrouve cette obstination tenace, et insulaire, à ne pas vouloir quitter la terre ferme, l'assise du réel, à refuser de confondre le dehors et le dedans, le haut et le bas, l'envers et l'endroit : en fait Guillevic tente de conjurer, et c'est en filigrane dans toute sa poésie, le vieux rêve d'osmose des surréalistes de réconcilier les contraires, comme le rêve et la réalité ou la veille et le sommeil. Le poète s'est expliqué à maintes reprises (bien qu'il ait été l'ami d'Eluard avec lequel il a partagé l'honneur d'être poète pendant la Résistance mais c'est une autre histoire) sur ses réticences envers le surréalisme qu'il réduira, non sans une certaine mauvaise foi, à du «Rimbaud scolastifié » - et auquel il n'adhéra jamais, parce que lui, n'a, selon ses propres termes, «jamais rien concédé au rêve ", dont il redoute les forces dissolvantes - et le mot "rêve » est à prendre au singulier et au pluriel. (Figure $4:$ : cylindre »).

Cette attitude, c'est celle du matérialiste à tout crin, du cartésien qui se méfie des images surtout lorsqu'elles sont comme chez les surréalistes, pur surgissement de 
l'inconscient et plus encore le fruit du hasard (objectif, évidemment), à la faveur duquel le poème se construit de proche en proche au fil d'associations, pour le moins surprenantes. Pour Guillevic, tout au contraire, le poème est le produit à la fois d'un jaillissement intérieur, d'une émergence et d'un contrôle, - celui du réel en premier lieu. Car ce qui le motive incessamment dans l'acte d'écrire, c'est de sommer le monde avec des mots (les titres des poèmes une fois de plus sont éloquents : Exécutoire), lui qui est en position d'être Requis par l'écriture.

\section{II - L'espace de l'écriture}

Guillevic, incessamment revient sur l'acte d'écrire : il faut à nouveau se référer aux titres des recueils qui, pour la plupart, ressortissent à l'écriture (quand le contenu du recueil lui-même n'est pas un art poétique): Creusement, Motifs, Encoches, Trouées, Fractures, Entailles, Impacts. Certains textes, comme Inclus, sont même entièrement consacrés au geste d'écrire, tendus autour de sa genèse, son élan, ses effets :

«Écrire,

C'est creuser dans du noir,

C'est au sein de ce noir

Y sacrifier.

Du noir qui est en soi

Le marier à du noir des mots.

(Inclus)

\section{cylindre}

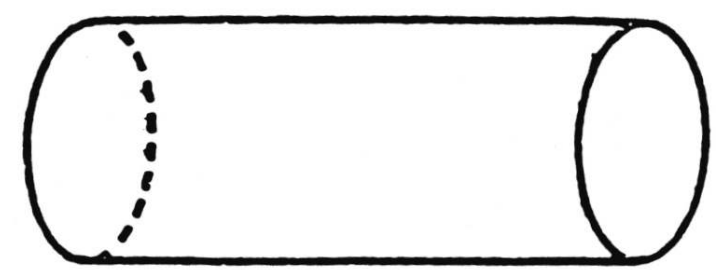

Si l'on quittait la sphère

Pour s'en aller ailleurs,

C'est à travers toi

Que l'on passerait.

J'imagine à peu près

Ce que ça pourrait être :

J'ai connu ta longueur

Dans tant de mauvais rêves.

Figure 4

On retrouve, - en fait on ne l'a jamais quitté -, le rôle de l'affrontement à la matière :

Écrire, -

Comme jusqu'ici

C'est-à-dire Affronter

Car les mots résistent : 


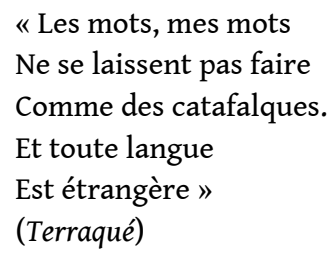

Les mots sont instruments de connaissance, et d'élucidation (et il faut prendre celui de «savoir» dans sa triple acception étymologique, qui renvoie à la connaissance, la saveur et la sagesse) :

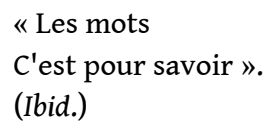

Mais aussi exorcisme :

"Quand tu regardes l'arbre et dis le mot tissu

Tu crois savoir et toucher même

Ce qui s'y fait (...)

Et la peur

Est presque partie.

(Exécutoire)

Les termes précis et tranchants comme une épure, abondent, qui disent l'acte d'entailler l'espace de la page avec les mots dans la circulation signifiante des blancs : "Écrire inscrire marquer graver garder" (Inclus). Mais ne nous y trompons pas, il ne saurait s'agir là de métaphores ${ }^{8}$ : la main qui écrit est celle d'un sculpteur affronté à la matière qu'il pétrit, modèle, élague, cisèle, pour nommer le monde tel qu'il est ${ }^{9}$ et le poème, « sculpture du silence $»^{10}$ est ce «monument hiératique » qui se dresse dans sa verticalité pour faire échec au temps :

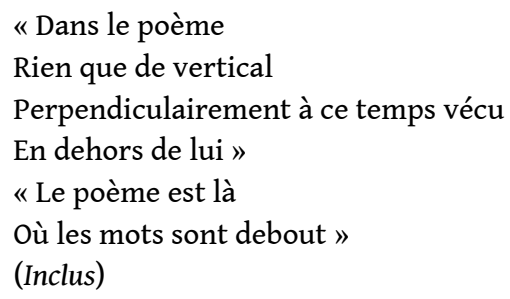

24 Mais le même danger que celui qui guette l'homme aux prises avec le monde, guette aussi l'artisan du poème aux prises avec la matière et l'espace du poème, et c'est celui pour l'écriture de se déliter, de s'effilocher : curieusement ce sont les adjectifs' ${ }^{11}$ qui menacent l'intégrité du poème, d'en trouer la trame compacte, salutairement ramassée sur elle-même :

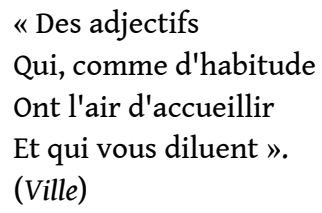

Comme on le voit, l'espace dans l'écriture guillevicienne est plus que jamais présent, non pas comme support de l'écrit, mais comme espace physique, tangible - à creuser, à cribler, espace cependant jamais comblé puisque l'écriture c'est «l'alliage obsédant /de plein et de vide » (Inclus) (on notera la technicité du terme que Guillevic préfère à celui, plus attendu, d'alliance), c'est "...la déréliction/Sur les pleins et dans les creux». (Étier). Les mots tracent des contours et des limites mais introduisent à la vacance habitée des blancs, à leur plénitude lacunaire qui ont valeur sémantique, rythmique, 
prosodique. Blanc « codé » pour Guillevic, qui en dit parfois "plus long que les mots » et qui, comme l'étier, - et la trouée sépare et réunit. Blanc fertile et poreux, lieu de passage et d'échange, éminemment franchissable, éminemment transgressable, il ouvre à d'autres trajets, « réseaux » « branchies » pour employer les mots de Guillevic. Je prendrais, en guise d'exemple ultime, le recueil Du Domaine, si éloquent à ce sujet (si l'on peut employer ce terme d'éloquence pour un poète qui pratique électivement la litote), tant par l'espace qu'il décrit que par celui qu'il dessine. Des suites brèves, (des "quanta " comme il les appelle et on notera qu'il s'agit d'un terme de physique, désignant des "quantités quantifiables ») qui, comportent chacune un vers ou deux, mangées de blancs et séparées par un tout petit rond, emblème minuscule mais hautement signifiant d'une poésie du fragment, insulaire en quelque sorte. Peut-être l'infiniment petit de la figure du cercle qui est l'une des figures les plus prégnantes de sa poétique. (Figure $5:$ « cercle»)

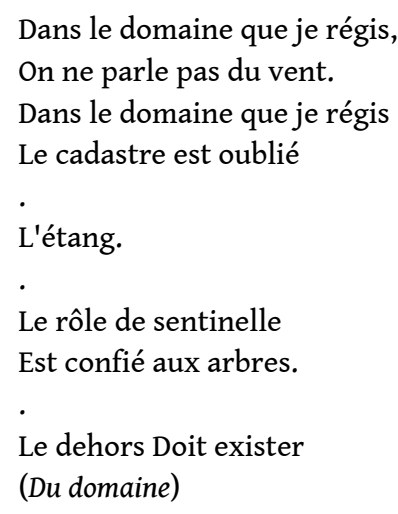

Le rôle du blanc est exemplaire dans cette poésie, qui figure, au sens le moins figuré du terme, un espace d'où les mots se sont absentés mais qui n'en continue pas moins à faire sens, - et rythme : il est non seulement la trame de l'écriture mais encore la trace de la traversée lyrique du poète qui pour emprunter d'autres voies que celle du je, n'est pas moins présent dans son poème : "Je est dépossédé-décentré-désoriginé, il n'est pas propriétaire, il n'a aucun pouvoir, il est ce lieu par où tout passe $»{ }^{12}$ On en revient à la figure de l'étier, emblématique du narcissisme de Guillevic: "Mon narcissisme concerne moins ma personne que ce qui se passe en moi, ce qui est - du moins à mes 
yeux - une expression du monde à travers moi. Ce qui m'intéresse en moi c'est l'étier $»^{13}$.

\section{cercle}

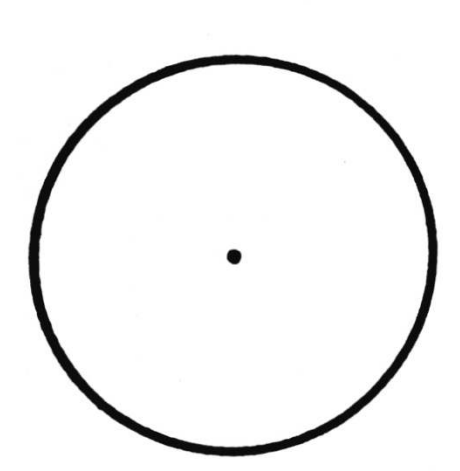

\section{Tu es un frère, On peut s'entendre. Fais-moi pareil, Enferme-moi. \\ Réchauffons-nous, Vivons ensemble Et méditons.}

Figure 5

\section{Conclusion}

Il est un mot à travers lequel je voudrais conclure, en ce qu'il me semble très exactement qualifier la poésie de Guillevic, dans son rapport à l'espace, et c'est celui de lapidaire. Un terme qui désigne, en tant que substantif, un «artisan qui taille, polit, grave les pierres précieuses", et qualifie en tant qu'adjectif un style "propre aux inscriptions gravées sur pierre » (et notamment aux inscriptions latines, remarquables pour leur concision). Guillevic ne cesse de le répéter : «Oui le vers [est] une langue tendue qu'on ne [peut] pas modifier, qu'on ne [peut] pas briser. Quelque chose de solide. De la nature de la pierre $»^{14}$

A la croisée de la sculpture, de la peinture, de la géométrie, de la musique, de l'architecture, l'écriture de Guillevic n'a de cesse de tracer, de circonscrire des contours qui tentent de préserver l'inviolabilité de son territoire. Un territoire tout entier replié autour de phrases brèves et compactes, sentences ou aphorismes porteurs de la sagesse et du savoir du monde. Au centre dans la plénitude de la figure de cercle ${ }^{15}$, exulte le poète porté comme à son insu par l'impersonnalité d'une parole qu'il a suscitée mais dont il n'est plus maître. 


\section{NOTES}

1. Toutes les références aux poèmes de Guillevic renvoie à l'édition Gallimard.

Cf. J.-M. Gleize: «centre glauque, le moi ouvert autour de l'étang fermé, fermé, ouvert, entrouvert », Littérature, oct. 79, $\mathrm{N}^{\circ} 35$, p. 85. Cf. également, parmi des multitudes d'exemples ce poème extrait de Terraqué, Poésie/Gallimard, pp. 76-77 : «Ce soir encore l'étang/Ne s'est pas mis debout/Au passage du vent./Les chambres sont glacées/Comme des carpes. La peur/Ne quitte plus les longs couloirs [/Et si l'étang se lève, libidineux,/Il n'aura pas raison de notre calme ;/Nos mains, qui caressent les femmes/Sauront l'atteindre et leur percer le ventre ».

2. «On parle souvent de toi comme un être de roc, un être primitif». Réponse de Guillevic: «C'est pour ne pas tomber dans un tellurisme mystique ». Vivre en poésie, Stock, 1980 p. 41.

3. Ibid., p. 32.

4. Ibid., p. 42.

5. Ibid., p. 52.

6. Ibid., p. 158-159.

7. Il faudrait parler aussi de l'écriture comme rite sacrificiel, comme rite du don, comme immolation («Il s'offre/Donc/Il écrit», Inclus) Et lorsqu'on sait que le sacrifice est le "moyen pour le profane de communiquer avec le sacré par l'intermédiaire d'une victime, c'est-à-dire d'une chose consacrée, détruite au cours de la cérémonie » (Hubert et Mauss, Mélanges d'histoire des religions, Paris, Alcan, 1929, cité par J. Cazeneuve dans Les rites et la condition humaine, PUF, 1958, p. 296), on mesure tout le sens que prendre le rapport entre la poésie et le sacré.

8. Cf. Vivre en poésie, op. cit., p. 182-183 : « La métaphore n'est pas pour moi l'essence du poème ; je procède par comparaison, non par métaphore. C'est une des raisons de mon opposition au surréalisme. Pour moi comme pour Jean Follain, une chose peut être comme une autre chose, elle n'est pas cette autre chose. On a dit [...] que la poésie moderne se reconnaît à ce qu'elle écarte le mot "comme". Je récuse cette définition. Je ne dis pas: "des lèvres de corail" car les lèvres ne sont pas en corail. Je dis naïvement "des lèvres comme du corail". Les mots s'insurgent d'être métaphorisés ! » et un peu plus loin, parlant de l'acte d'écrire, il ajoute : Je procède «comme si j'étais un carrier et que je creusais dans une falaise à pic - cette falaise étant à la fois le langage et la chose ».

9. On ne trouve, en effet, nul désir chez Guillevic, comme chez les surréalistes par exemple, de changer le monde; il ne s'agit pour lui que de le nommer: «Le retour du réel passe; entre autres, par ce geste-là : de la réduction des choses à elles-mêmes ». (J.-M. Gleize, Poésie et figuration, Seuil, 1983, p. 202).

10. Vivre en poésie, op. cit., p. 186 : «C'est précisément cette inclusion du silence dans les mots qui distingue le poème de la prose. La difficulté est de faire entendre le silence, de le faire sentir. Je dirais même de le faire toucher. J'ai essayé dans maints poèmes ».

11. Cf. également la position de Guillevic sur l'adjectif dans Choses parlées (Ed. Champ Vallon, 1982, p. 75) : «L'adjectif est un trou dans le tissu, c'est un vide. »

12. J.-M. Gleize, op. cit., p. 85.

13. Vivre en poésie, op. cit., p 238. cf. également, p. 220 : «Et puis, le poète n'est-il pas l'étier qui reçoit ce qu'il peut du monde et en garde ces petits tas de sel : les poèmes? ".

14. Vivre en poésie, op. cit., p. 30.

15. « La précieuse clôture du cercle, si instamment appelée, est celle de la méditation, c'est-à-dire de cette rare espèce de pensée qui ne disperse pas son énergie, mais transforme ses propres dépenses en aliment.», Frédérique Martin-Scherrer : "Figurer, lecture d'Euclidiennes », Revue Sud, « Guillevic, Les chemins du poème », Hors série, 1987, p. 123. 


\section{AUTEUR}

\section{COLETTE GUEDJ}

Université de Nice-Sophia Antipolis 